http://jmscr.igmpublication.org/home/ ISSN (e)-2347-176x ISSN (p) 2455-0450 crossref DOI: https://dx.doi.org/10.18535/jmscr/v7i8.126

Journal Of Medical Science And Clinical Research

\title{
Study of clinical outcome of laser peripheral iridotomy in patient with spectrum of primary angle closure
}

\author{
Authors \\ Manjula Y.M ${ }^{1}$, Chethana B.S. ${ }^{{ }^{*}}$
}

${ }^{1}$ Associate professor, Department of Ophthalmology, BGS Global institute of Medical sciences, Bangalore

${ }^{2}$ Senior Resident, Department of Ophthalmology, BGS Global institute of Medical sciences, Bangalore *Corresponding Author

Chethana B.S.

Senior Resident, Department of Ophthalmology, BGS Global institute of Medical sciences, Bangalore, India

\begin{abstract}
Aim: To evaluate the outcome of peripheral laser iridotomy as first line of management in patient with spectrum of primary angle closure, which includes primary angle closure suspect (PACS), primary angle closure (PAC) and primary angle closure glaucoma (PACG).

Material and Methods: In our study prospective analysis of 30 patients with spectrum of primary angle closure visited to BGS Global institute of Medical sciences, Department of ophthalmology glaucoma clinic, were evaluated by one glaucoma consultant using slit lamp, goniolens and treated with Nd-Yag laser peripheral iridotomy as first line of management. Depth of peripheral anterior chamber assessed by slit lamp using van Herick technique. intraocular pressure measured using Goldman applanation tonometer. Angle was assessed using sussman 4 mirror goniolens by shaffers grading. Patients were followed up for 6month post laser peripheral iridotomy.

Result: In our study conducted for 1 year from March 2017 to March 2018 and one year follow up, 30 patient with spectrum of primary angle closure, which included primary angle closure suspect (PACS) 4 patient, primary angle closure (PAC) 24 patient and primary angle closure glaucoma(PACG) 2 patient were included. All patient underwent peripheral laser iridotomy as first line of management, intraocular pressure (IOP) was less than $16 \mathrm{mmof} \mathrm{Hg}$ in 28 patients, 2 patients IOP was more than $20 \mathrm{~mm}$ of $\mathrm{Hg}$ was started on medical management timolol0.5\% BID. Peripheral angle depth increased in all patients which were assessed by slit lamp. Using van Herrick technique. angle evaluation was done using sussman goniolens reveled open angle after peripheral laser iridotomy in 28 patients. Except two patients angle remained closed because of synaechial angle closure.

Conclusion: Out of 30 patients with spectrum of primary angle closure treated with peripheral laser iridotomy as first line of management, all patient had increases in peripheral anterior chamber depth, 28 patient had IOP less than 16 mmof hg and open angle.only two patient angle remained closed with IOP more than $20 \mathrm{~mm} \mathrm{Hg}$ because of synaechial angle closure. Which was managed by medical treatment, So peripheral laser iridotomy is the procedure of choice for first line of management in patient with spectrum of primary angle closure.

Keywords: spectrum of primary angle closure, primary angle closure suspect (PACS), primary angle closure (PAC), primary angle closure glaucoma (PACG), laser peripheral iridotomy.
\end{abstract}




\section{Introduction}

Glaucoma is a progressive disease entity which comprises of specific visual field changes corresponding to characteristic optic disc changes, which is intraocular pressure (IOP) sensitive ${ }^{1}$.

Glaucoma is the second leading cause of world preventable blindness after cataract and accounts for $15 \%$ of global blindness. Asians represent $47 \%$ of those with glaucoma and $87 \%$ of those with angle closure glaucoma $(\mathrm{ACG})^{2}$. Primary angle closure glaucoma (PACG) has been reported to be more prevalent in south East Asian countries than rest of the world. The proportion of all cases suffering from significant loss of vision is three times higher in PACG than POAG as considerable damage can occur before symptoms become apparent ${ }^{3}$.

Primary angle closure diseases (PACD) is a group of disorders characterized by a shallow anterior chamber with predisposition to appositional or synechial closure of the anterior chamber angle leading to raised intraocular pressure and subsequent optic neuropathy. spectrum of primary angle closure, includes primary angle closure suspect (PACS), primary angle closure (PAC) and primary angle closure glaucoma (PACG).

Laser peripheral iridotomy is the standard first line intervention in both acute and chronic forms of primary angle closure glaucoma. It is successful in preventing recurrence of acute attacks and virtually eliminates the risk of an acute attack in the fellow eye. The mechanism of action is relief of relative pupillary block, allowing the convex iris to flatten and widening of the anterior chamber angle ${ }^{4}$.

It is essential to evaluate the response to LPI by studying changes in anterior segment morphology. Advances in anterior segment imaging have allowed for quantitative and reproducible measurement of the parameters. Ultrasound biomicroscopy and anterior segment optical coherence tomography are more preferred modalities of anterior chamber parameter measurement. There are many studies which have documented change in anterior chamber anatomy post LPI. In our study we evaluated before and after peripheral laser iridotomy peripheral anterior chamber depth using slit lamp Van Herick technique, intraocular pressure measured using Goldman applanation tonometer. Angle was assessed using sussman 4 mirror goniolens by shaffers grading.

\section{Material and Methods}

30 patients with spectrum of primary angle closure diseases visited to BGS Global institute of Medical sciences, Department of ophthalmology glaucoma clinic were analyzed prospectively from March 2017 to March 2018 and one year follow up before and after laser peripheral iridotomy as first line of management. They were followed for 1year. Ethical committee clearance from the institution was obtained .Informed consent was obtained from all the patient.

All patients underwent evaluation by one glaucoma consultant includes;

1. Medical and ophthalmic history

2. Best corrected visual acuity measured

3. Anterior segment evaluation to rule out secondary angle closure.

4. Peripheral anterior chamber depth assessed by slit lamp using van Herick technique before and after Nd-Yag laser peripheral iridotomy

With an optical section of the limbal cornea, slit beam angulated to 60 degree width of the corneal section compared to width of the shadow adjacent to it.

1:1- open angle, $\mathrm{VH}$ grade 4

1:1/2- open angle, $\mathrm{VH}$ grade3

1:1/4- narrow angle, $\mathrm{VH}$ grade2 (Angle closure possible)

$1:<1 / 4$ - angle closure likely, VH grade1.

5. Intraocular pressure measured by Goldmann applanation tonometry.

6. Gonioscopic grading of anterior chamber angle by sussman lens ${ }^{5}$ by Shaffer grading before and after Nd-Yag laser peripheral iridotomy 


$\begin{array}{lll}\text { ACD } & \text { Angle status } & \text { visible structures } \\ 0 & \text { Closed } & \text { no structures visible } \\ 1 & \text { Extremely narrow } & \text { schwalbes line } \\ 2 & \text { Narrow } & \text { trabecular meshwork } \\ 3 & \text { open } & \text { sclera spur. } \\ 4 & \text { wide open } & \text { ciliary body. }\end{array}$

Non visibility of the pigmented trabecular meshwork in $180^{0}$ or more of angle circumference in primary position on gonioscopy. (performed in dark room with narrow $1 \mathrm{~mm}$ slit beam) is taken as occludable angles and labeled as PACD.

7. Stereoscopic examination of the optic nerve head with 78 dioptre lens.

The participants were classified into PACS, PAC and PACG groups according to the International Society for Geographical and Epidemiological Ophthalmology guidelines ${ }^{6}$.

The subjects who fulfilled the diagnostic criteria of primary angle closure spectrum were included in the study.

Patients with co-existent retinal pathology, previous intraocular operations, ocular trauma, uveitis and patients with secondary angle closure glaucoma were excluded from the study.

One drop of $2 \%$ pilocarpine was instilled to the eyes for about 4 times, one hour prior to LPI. The Q-switched Nd:YAG laser (1064nm) and Abraham lens with normal saline as coupling agent was used. The following setting was applied for laser iridotomy: 2-3 shots/burst using energy $1-3 \mathrm{~mJ} / \mathrm{burst}$ to the treatment site. Patency of the iridotomy was confirmed by the gush of aqueous through iridotomy from posterior to anterior chamber along with pigment dispersion. After LPI, all patients received $1 \%$ prednisolone acetate ophthalmic suspension or $0.1 \%$ dexamethasone 4 times a day for 4 days. Brimonidine $0.2 \%$ eye drops BID for 4days. None of the patients showed spike in intraocular pressure, measured after one hour of LPI.

Patients were followed on $1^{\text {st }}$ day, 1 week, 1 month, 3 month, 6month and 1year after peripheral laser iridotomy.

No complication of laser was noted during follow up till 1year.

\section{Result}

1. The 30 patients who fulfilled the above mentioned inclusion criteria were enrolled in the study. The patients were divided into PACS, PAC and PACG groups which included 4, 24 and 2 respectively

\begin{tabular}{|l|c|}
\hline PACS & 4 \\
\hline PAC & 24 \\
\hline PACG & 2 \\
\hline PACD & 30 \\
\hline
\end{tabular}

2.Male to female ratio was 4:26.

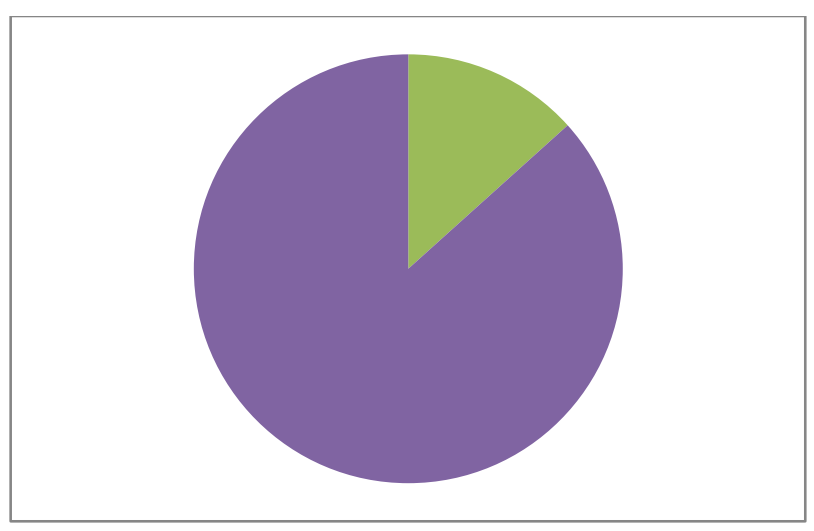

PACD was common in females in our study

3. Spectrum of primary angle closure was more commonly after 40years of age group.

\begin{tabular}{|l|c|c|}
\hline AGE & FEMALE & MALE \\
\hline 10-20years & & \\
\hline 21-30years & & \\
\hline 31-40years & 2 & \\
\hline 41-50years & 15 & 2 \\
\hline 51-60years & 9 & 2 \\
\hline
\end{tabular}

4. All 30 patients who had peripheral shallow anterior chamber before iridotomy had increases in peripheral anterior chamber depth after laser peripheral iridotomy

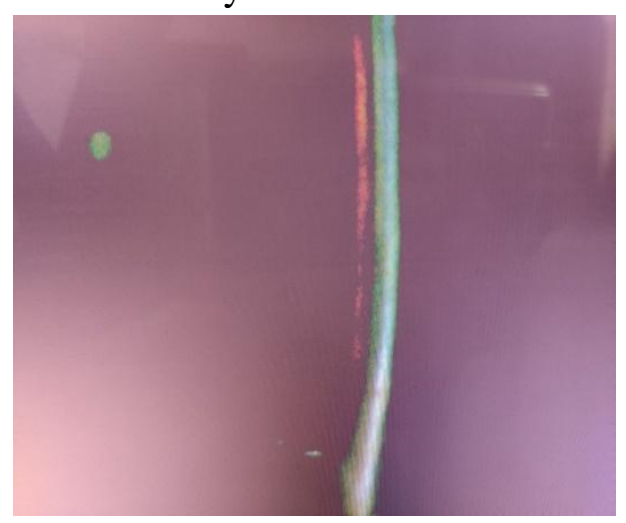

ACD before laser iridotomy. 


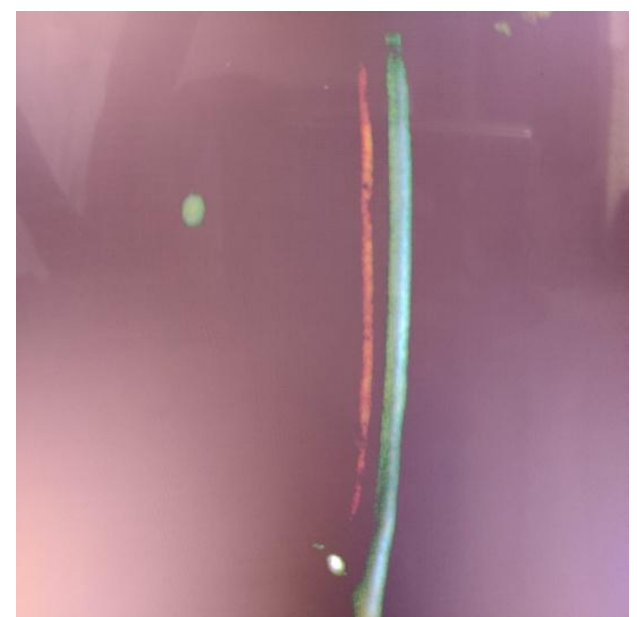

ACD after laser iridotomy.

5. out of 30 patients with PACD all patient who underwent peripheral laser iridotomy as first line of management , 28 patient had IOP less than $16 \mathrm{mmof}$ hg and open angle .only two patient angle remained closed with IOP more than $20 \mathrm{~mm}$ $\mathrm{Hg}$ because of synaechial angle closure. 2 patient IOP was more than $20 \mathrm{~mm}$ of $\mathrm{Hg}$ w as started on medical management timolol $0.5 \%$ BID. So peripheral laser iridotomy is the procedure of choice for first line of management in patient with spectrum of primary angle closure

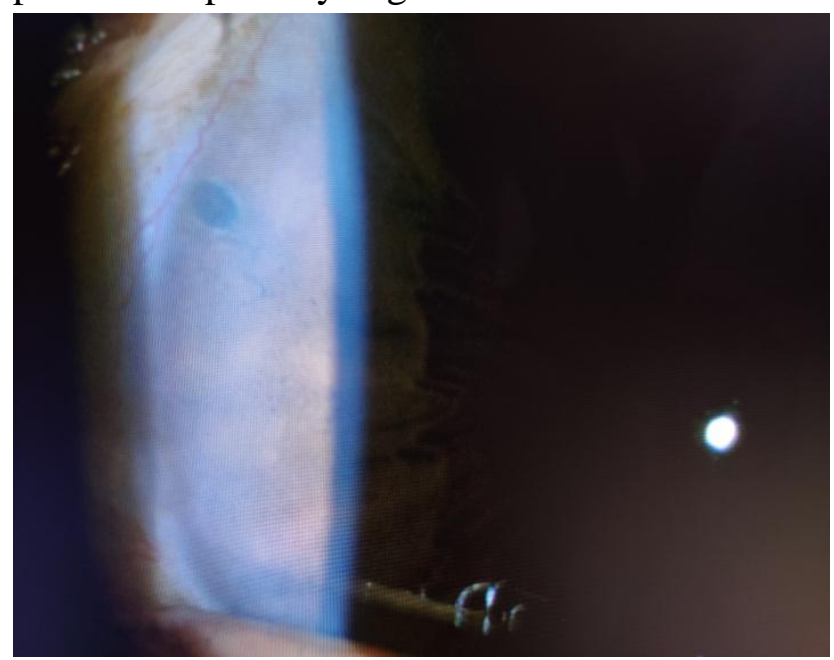

Patent peripheral laser iridotomy

\section{Discussion}

Laser peripheral iridotomy is the first line of treatment in the management of eyes with narrow angles. It is known that iridotomy relives relative pupillary block by providing an alternate pathway for the aqueous humor to drain from the posterior to anterior chamber angles ${ }^{7}$.
The Van Herrick method is a non-invasive and simple outpatient procedure in assessing anterior chamber depth. However, it is imprecise and subjective. We have used the Van Herrick technique in our study done by one glaucoma consultant.

Assessment of angles by gonioscopy involves enumerating the posterior most angle structure visible in the angle, and the angle between the peripheral cornea and iris. Though more precise than the Van Herrick technique, gonioscopy is also associated with inter and intra observer variability $^{8}$ in our study done by one glaucoma consultant.

Advances in anterior chamber imaging, anterior segment optical coherence tomography and imaging modalities Rotating Scheimpflug camera and lenstar have allowed for quantitative and reproducible measurement of anterior segment parameters .but instrument are costly. Which was not available in our institute.

In the study conducted by Lopez-Callabero et al, they observed significant deepening of CACD after LPI in patients with narrow angles and PACG using Rotating Scheimpflug camera ${ }^{9}$. Talajic et al concluded in their study that although an increase in CACD was noted after LPI, it was statistically insignificant among patients with PACS $^{10}$. Grewal et al in their study concluded that extent of the LPI-induced peripheral anterior chamber depth increase was enhanced with increasing distance from the optical axis but no change occurred in $\mathrm{CACD}^{11}$.

In our study, 30 patient with spectrum of primary angle closure, which included primary angle closure suspect (PACS) 4 patient, primary angle closure (PAC) 24 patient and primary angle closure glaucoma (PACG) 2 patient. All patient who underwent peripheral laser iridotomy as first line of management, intraocular pressure (IOP) was less than $16 \mathrm{~mm}$ of $\mathrm{Hg}$ in 28 patient 2 patient IOP was more than $20 \mathrm{~mm}$ of $\mathrm{Hg}$ w as started on medical management timolol $0.5 \%$ BID. Peripheral angle depth increased in all patients who were assessed by slit lamp using van Herrick 
technique. angle evaluation was done using sussman goniolens reveled open angle after peripheral laser iridotomy in 28 patients. Except two patient angle remained closed because of synaechial angle closure.

\section{Conclusion}

Primary angle closure glaucoma is one of the common types of glaucoma affecting over 15 million individuals worldwide. The number of PACG cases projected to reach 21 million by 2020 .it is one of the preventable blindness if treated early it can be treated by medical management, laser and surgery. In our study out of 30 patients with spectrum of primary angle closure treated with peripheral laser iridotomy as first line of management, all patient had increases in peripheral anterior chamber depth, 28 patient had IOP less than $16 \mathrm{mmof}$ hg and open angle .only two patient angle remained closed with IOP more than $20 \mathrm{~mm} \mathrm{Hg}$ because of synaechial angle closure. So peripheral laser iridotomy is the procedure of choice for first line of management in patient with spectrum of primary angle closure. The limitation of our study was lack of long term follow up.

\section{References}

1. Sihota R, Tandon R .Parson"s diseases of the eye .21 st edition. Elsevier;2011:280 .

2. Khandelwal R, Raje D, Rathi A, Khandelwal R. EVALUATION OF NARROW ANGLE GLAUCOMA CASES ATTENDING A TERTIARY CARE CENTER OF CENTRAL INDIA. Journal of Evidence Based Medicine and Healthcare. 2015;2(43):7724-31.

3. Ahram DF, Alward WL, Kuehn MH. The genetic mechanisms of primary angle closure glaucoma. Eye. 2015;29(10):1251-9.

4. Grewal SP, Jain R, Grewal D. Evaluation of anterior segment changes following laser peripheral iridotomy using Pentacam
Scheimpflug imaging system in eyes with primary angle closure (PAC). Highlights of ophthalmology.2008;36(4):13-14

5. Singh P, Tyagi M, Kumar Y, Kuldeep K, Sharma P. Gonioscopy: A Review. Open Journal of Ophthalmology. 2013;03(04): 118-121.

6. Foster PJ, Buhrmann R, Quigley HA, Johnson GJ. The definition and classification of glaucoma in prevalence surveys. British journal of ophthalmology. 2002;86(2):238-42.

7. He M, Friedman DS, Ge J, Huang W, Jin C, Lee PS, Khaw PT, Foster PJ. Laser peripheral iridotomy in primary angleclosure suspects: biometric and gonioscopic outcomes: the Liwan Eye Study. Ophthalmology. 2007;114(3):494-500.

8. Pakravan M, Sharifipour F, Yazdani S, Koohestani N, Yaseri M. Scheimpflug imaging criteria for identifying eyes at high risk of acute angle closure. Journal of ophthalmic \& vision research. 2012;7(2):111-7.

9. Lopez-caballero C, Puerto-Hernandez B, Munoz-Negrete Fj, Rebolleda G, Contreras I,Cabarga C,Corral A. Quantitative evaluation of anterior chamber depth changes anterior chamber using pentacam anterior segment analyzer .European journal of ophthalmology .2009:20(2):327-32.

10. Talajic JC, Lesk MR, Nantel-Battista M, Harasymowycz PJ. Anterior segment changes after pilocarpine and laser iridotomy for primary angle-closure suspects with Scheimpflug photography, journal of glaucoma 2013:22(9):776-9.

11. Jain R, Grewal D, Grewal SP. Quantitative analysis of anterior chamber following peripheral Laser iridotomy using pentacam in eyes with primary angle-closure. European journal of ophthalmology. 2012:14:0.doi:10.5301/ejo.5000158. 